\title{
Experimental and Analytical Study of Torsional Vibration under Multiaxial Loading in Time Domain
}

\author{
Dionísio José Rodrigues da Costa ${ }^{1,2}$, Ladário da Silva ${ }^{1,3}$, Jorge Rodrigues Durán ${ }^{1,4}$ and José Flávio Feiteira ${ }^{4}$ \\ 1. Programa de Pós-graduação em Engenharia Metalúrgica (PPGEM), Escola Industrial Metalúrgica de Volta Redonda (EEIMVR), \\ Universidade Federal Fluminense (UFF), Volta Redonda 27255-125, RJ, Brasil \\ 2. Posto Regional de Polícia Técnico-Cientifica de Volta Redonda (PRPTC-VR), Volta Redonda 27180-000, RJ, Brasil \\ 3. Departamento de Física, Instituto de Ciências Exatas (ICEx), UFF, Volta Redonda 27213-145, RJ,Brasil \\ 4. Departamento de Engenharia Mecânica, EEIMVR, UFF, Volta Redonda 27255-125, RJ, Brasil
}

\begin{abstract}
The torsional vibration of power transmission shaft is a phenomenon whose analytical modeling can be represented by a differential equation of motion proposed by technical literature. The solutions of these equations need coefficients and parameters that, usually, must be experimentally estimated. This work uses a resistive electric SG (strain gage) to dynamically determine strains produced in the shaft due to harmonic oscillatory motion under multiaxial loading. This movement is simulated on a prototype specially developed for this purpose. It comprises a pulley attached to the end of a stepped cantilevered shaft, which is clamped at the opposite end. In this configuration, a cam generates a torque to the system, springs regulate the stiffness and the damping coefficient of the assembly, as well as they can be suitably adjusted to produce an underdamped condition. The main advantage, highlighted in this study, refers to a major simplification. Although the system under study shows multiple degrees of freedom (torsion and bending), the shape and the positioning of linking SGs with the resistor bridge (Wheatstone Bridge), allow to evaluate the loading effects independently, as if only one degree of freedom of the system exists at a time domain. Strains graphs for two forms of cyclic torsional oscillation, analytical and experimental, were successfully generated.
\end{abstract}

Key words: Underdamped system, free and forced response, torsional vibration, strain gauges.

\section{Introduction}

The analysis of the vibration control considers any element that produces or suffers a form of repetitive dynamic effort. Currently, this analysis is widely applied in the mechanical industry and it is also used in preventive condition, which includes a continuous monitoring of the element of interest and in corrective condition. The dynamical strength analysis, very applied in the machining area, requires considerations of the cyclical fluctuations that are intrinsically related to any mechanical element that rotates. The interest in increasing the quality of a particular product, in order to avoid failures of any kind, to improve efficiency and to reduce costs, boosts the interest to a constant

Corresponding author: Dionísio José Rodrigues da Costa, Ph.D. student, research fields: vibration, dynamic strain analysis, fatigue, and prototype design. improvement in the analysis of strength and vibration of rotating elements. Therefore, it is worth citing some studies in this subject. Wu [1] has numerically analyzed the vibration of a variable section shaft using the Lagrangian method. Chen [2] has investigated the torsional vibrations of a cylinder with varying cross section. Castro et al. [3] have investigated the effects of the correction in the dynamic forces of measurements performed with piezoelectric dynamometer. Quin et al. [4] have studied the propagation of the torsional vibration in the shaft material using transformation matrix and finite elements. Gaspari et al. [5] used a model of boundary elements to analyze the coupled torsion. This study provides the complete shear stress field by evaluating properties of the polygonal cross section. In this investigation, a numerical model allows an analysis of 
performances of the Saint-Venant model. Huang [6] has studied the characteristics of torsional vibration of an unbalanced rotor by numerical simulation. In his study, the torsional vibration of the shaft can result in lateral vibration with bisynchronous frequency. Especially, when the rotating frequency is near the natural torsional frequency, where torsional vibration is stronger, the bisynchronous vibration becomes rather strong. Han et al. [7] have made the dynamic analysis of a geared rotor-bearing system with a breathing slant crack. They adopted the finite element model of a geared rotor with slant crack. The comparative study with slant cracked geared rotor is carried out to explore distinctive features in their modal, parametric instability and frequency response behaviours. Saka et al. [8] have studied the torsional vibration produced by a shaft with a cam subjected to a force. In this study, the obtained results were based only on the numerical analysis, and it does not apply to an experimental procedure. In the work of Xiang et al. [9], a laser torsional vibrometer was used to measure the torsion vibration of a rotating shaft system under electrical network impact. The system is modelled as a chain consisting of an elastic spring with concentrated mass points, and the multi-segments lumped mass model is established for this shafting system. The torsional vibrations of the shaft system were measured under electrical network impacts in laser Doppler torsional vibrometer. Salarieh et al. [10], in their paper, have analyzed the free vibration of a cantilever. Roukema et al. [11] have introduced a time domain model of the torsional-axial chatter vibrations in drilling. The model takes into account the exact kinematics of rigid body, and the coupled torsional and axial vibrations of the drill. The tool is modeled as a pretwisted beam that exhibits axial and torsional deflections due to torque and thrust loading. In the present work, torsional vibration monitoring techniques were applied, so that the strains were monitored at a given point on the surface of the shaft under stress in the elastic range and in the time domain. The main advantage highlighted in this study refers to an interesting simplification. Based on the condition that the system under study, although having several degrees of freedom (torsion and bending), the shape and connection position of SGs with the bridge resistance (WB) allowed to evaluate the deformation produced by each type of multiaxial loading effort, considering a system of one degree of freedom for each component of the multiaxial strain. Starting from this premise, SGs (strain gages) were fixed on the prototype shaft, designed and built specifically for this purpose, whose deformation point, located on the surface, has been converted into analog signal and transmitted directly by a static contact. This analysis considers both free and forced vibrations, with damping. Free vibrations are described when the pulley has a torsional displacement and the shaft is moved from its equilibrium position, allowing the shaft to vibrate freely. In the case of forced vibration, an external force, due to the influence of the cam, cyclically excited the pulley and shaft. This study carried out two forms of analysis involving, each one, one degree of freedom using a prototype, which simulated the cyclic torsional vibration in a stepped shaft section, subjected to a certain CE (condition of effort). Many different forms of damping coefficients produced by regulating the prototype springs may influence such CE.

\section{Experimental Setup}

Based on this regulation prototype, a prototype, that could undergo underdamped vibratory motion, was conceived, as shown in Figs. 1a and $1 \mathrm{~b}$.

The prototype was basically constituted by a counterweight, a pulley, a shaft and an electric motor of DC (direct current). The DC motor is coupled to a cam that is associated with a lever and a set of springs, in an organized manner. This yielded a final setup of easy control and low commercial cost. Its general arrangement is showed in Fig. 1. In this prototype, the end of the stepped shaft is fixed in a pulley whose 


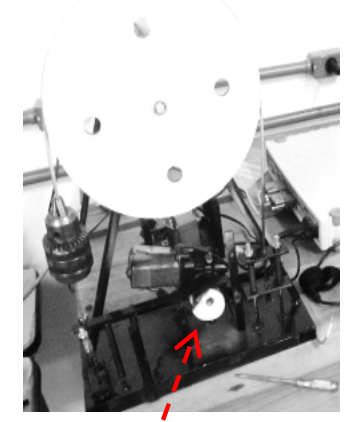

(a) Partial cam

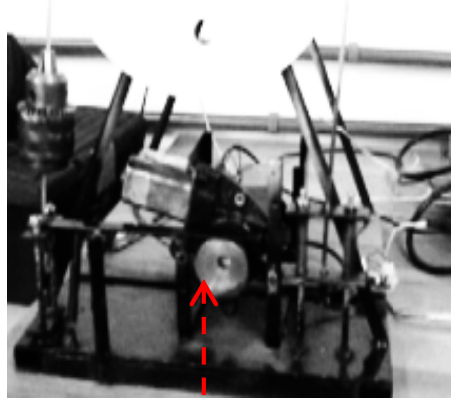

(b) Full cam
Fig. 1 General set of prototype assembly with (a) partial and (b) full cam.

function is to cause the torsion of the shaft in cyclical oscillatory movements. This mechanical element is rotated by using in one side a fixed cable, a counterweight and a spring, and in the other side, a set of springs. Once established the continuous and cyclic movements of the mechanical system by forcing the drive, a damped oscillatory motion is produced, providing a free torsional vibration when it is applied a partial cam (Fig. 1a) and forced torsional vibration when it applied a full cam (Fig. 1b), keeping the same physical characteristics of the joint.

The chosen SGs are transducers of the resistive type, uniaxial, from the KYOWA brand, $120 \Omega$, GF2.12. A data conditioner, manufactured by HBM, Spider 8-SR-30 model, was used to capture the analogic signals from SGs, turning them into digital signals. The electric interconnection of SGs was made by a WB (Wheatstone Bridge) circuit. The measures were accessed in real time by a Catman Easy 3.0 software, which allowed the acquisition of digital data. In all experiments, the initial parameters, the displacement of the counterweight $\left(x_{0}=0\right)$ and the initial velocity $\left(v_{0}=\right.$ 0 ) were fixed. Using a cam (partial/complete), fixed to the motor electric shaft, according to Fig. 1, torsions are produced successively, in cyclic movements, yielding strains in the SGs (Fig. 2). Analysis of such strains made it possible to evaluate the influence of the dynamics of boundary condition. Two SGs, connected to the spider $(1 / 2 \mathrm{WB})$, allowed the evaluation of the shaft strain imposed by the pulley oscillation, as shown

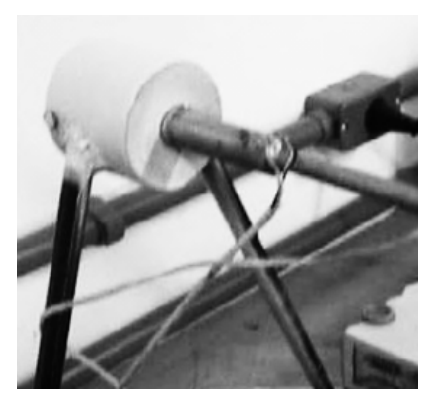

Fig. 2 SG torsional position.

Table 1 General electrical arrangement of strain.

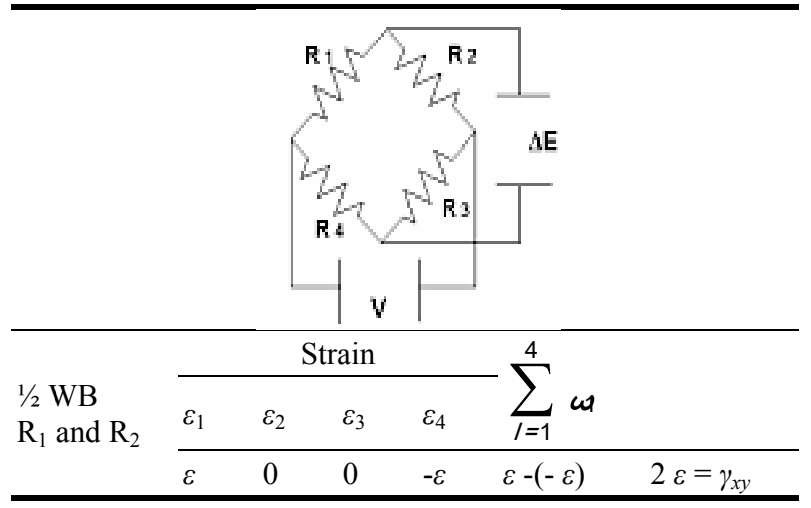

in Table 1. Built this way, this prototype mimics very accurate torsional vibration, allowing a great variety of studies like overdamped and critically damped motion.

\section{Analytical Model}

In the analytical study, a specific program written in Matlab language is used. This program simulates the movement of the cyclic behaviour, i.e., it twists the shaft and then frees it. It considers a shaft with a fixed end, while the other end undergoes the twist. In order to select the SGs properly, it is necessary to estimate the maximum deformation of the shaft previously to which the SGs are connected. The torsion intensity depends on the shaft stiffness $\left(K_{\text {res }}\right)$ and on the rigidity of the assembly $\left(K_{r}\right)$, as shown in Fig. 3. $K_{\text {res }}$ and $K_{r}$ are defined by Eqs. (1) and (2), respectively.

$K_{\text {res }}$, is the stiffness of the stepped shaft with three distinct sections, defined by Eq. (1):

$$
K_{\text {res. }}=(k 4 \cdot k 5 \cdot k 6) /(k 4+k 5+k 6)
$$

and $k_{r}$, the final assembly stiffness, Eq. (2)

$$
K_{r}=k 1+k 2+k 3+k_{r e s}
$$




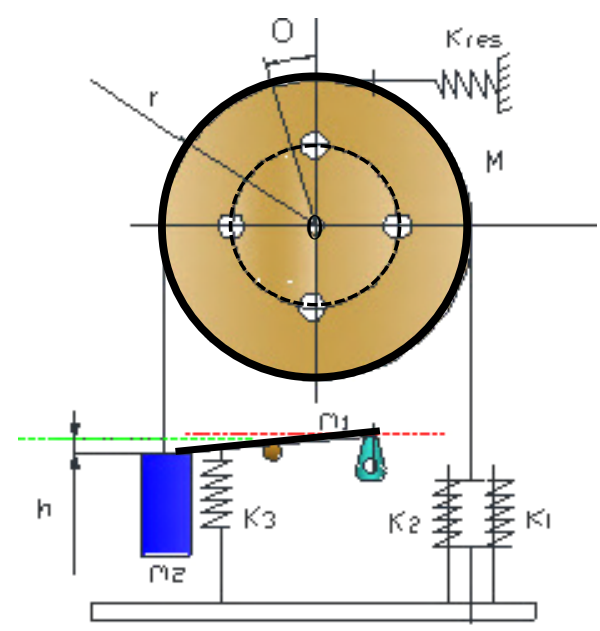

Fig. 3 Scheme of the stiffness distribution.

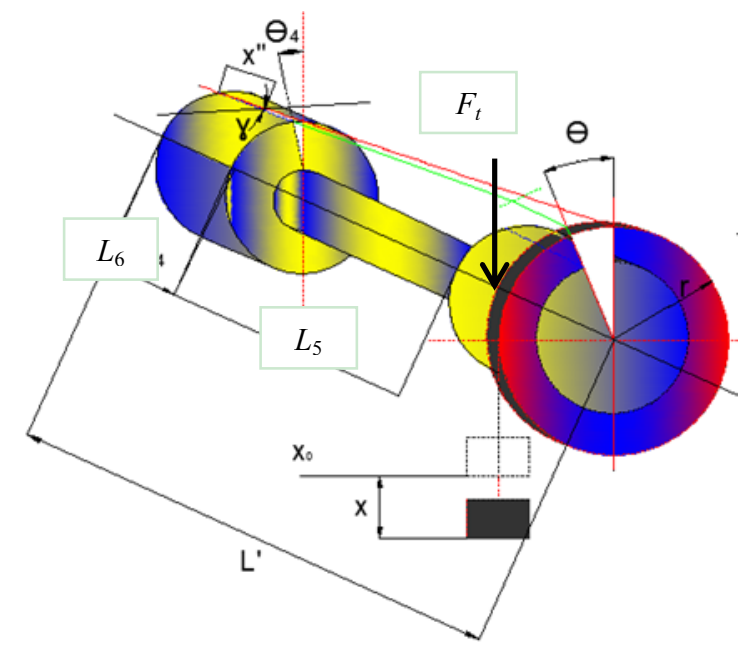

Fig. 4 Angular variation strain on shaft $\left(\theta_{4}(t), \theta(t)\right)$.

The simplified adopted model, shown in Fig. 4, allowed to separately analyze the strains imposed on the shaft based on the initial boundary conditions.

In Fig. 4, $x$ is the vertical displacement of the counterweight. $x^{\prime \prime}$ is the distance from the grip point to the strain gages location point. $\theta$ is the twist angle of the section. $\gamma$ is distortion angle, i.e., the relative angle of twist. $\gamma_{\max }$ is the maximum distortion angle. $L$ ' is the total length of the shaft. $r$ is the radius of the pulley. $\theta_{4}$ is the deformation of the length section $L_{6}$.

The torsion force $F_{t}$ is responsible for twisting the pulley within a certain angle $(\theta)$, described by Eq. (4). Due to the vertical displacement of the counterweight position $x_{0}=0$ to the position $x_{0}=x$, a strain arises in the shaft. Such strain results in a distortion angle $(y)$ in the gages location point. This system analysis highlighted two types of strain shaft. The first one occurs due to the shaft deflection. The second one arises due to the twisting of stepped shaft. The $\operatorname{arc}(\theta(t))$ at the shaft end (Fig. 3) is related to the displacement $(x(t))$, according to Eq. (3):

$$
\theta(t)=\frac{x(t)}{r}
$$

The maximum angle $\left(\theta_{\max }\right)$ of the shaft is given by Eq. (4):

$$
\theta_{\max }=\int_{0}^{1} \frac{T_{\max }(l)}{G J(x)} d l+c_{1}
$$

The torsion angle $(\theta)$, already defined in Eq. (3), can be related with the tangential force $F_{t}(r)$ and the shaft transversal elastic modulus, as shown in Eq. (5). The distortion angle $(8)$ is defined in Eq. (6).

$$
\begin{array}{r}
\theta(t)=\frac{F_{t}(t) \cdot r}{\mathrm{G}}\left(\frac{l_{4}}{J_{4}}+\frac{l_{5}}{J_{5}}+\frac{l_{6}}{J_{6}}\right) \\
\gamma(\mathrm{t})=\frac{\mathrm{F}_{\mathrm{t}}(\mathrm{t}) \times \mathrm{r}}{\mathrm{G}}\left(\frac{\mathrm{r}_{6}}{\mathrm{~J}_{6}}+\frac{\mathrm{r}_{5}}{\mathrm{~J}_{5}}+\frac{\mathrm{r}_{4}}{\mathrm{~J}_{4}}\right)
\end{array}
$$

The radius $r_{4}, r_{5}$ and $r_{6}$ are the prototype sections shaft radius. The inertial moments $J_{4}, J_{5}$ and $J_{6}$ are the corresponding shaft section inertial moments. The tangential force $F_{t}(r)$, used in Eq. (5), is now related to the vertical displacement and defined in Eq. (7).

$$
F_{t}(t)=\frac{x(t) \cdot G}{r^{2}\left(\frac{l_{4}}{J_{4}}+\frac{l_{5}}{J_{5}}+\frac{l_{6}}{J_{6}}\right)}
$$

The lengths $l_{5}$ and $l_{6}$ are clearly shown in Fig. 4 . The length $l_{4}$ is visible, for better clarity, in Fig. 6 .

Thus, the torsion angle $\left(\theta_{4}\right)$, shown in Fig. 4 , can be related to the tangential force $F_{t}(r)$ according to Eq. (8).

$$
\theta_{4}(t)=\frac{F_{t}(t) \cdot r}{G}\left(\frac{l_{6}}{J_{6}}\right)
$$

In general, the SG deformations are sufficiently small, so that one can consider the condition $\theta_{4} \cong \sin \left(\theta_{4}\right)$, which is true for very small angles.

There is a geometric relationship between the displacement of the counterweight and the angular 
pulley rotation. The balance oscillates vertically, while the pulley swings angularly. In static equilibrium condition, the cable is tensioned by a force $\left(F_{t}(t)\right)$, which is other possible way to define $\left(F_{t}(t)\right)$, done in Eq. (9):

$$
F_{t}(t)=K_{r} \cdot x(t)
$$

where, $\mathrm{K}_{r}$ was already defined in Eq. (2). In order to establish the balance equation of motion, it was considered the Newton and the Euler relations in $\mathrm{x}$ direction, shown in Eqs. (10) and (11).

$$
\begin{gathered}
F t . r-K_{r}\left(x-x_{0}\right) \cdot r=I \ddot{\theta} \\
\operatorname{m}^{\prime}{ }^{2} \ddot{\theta}-\mathrm{K}_{\mathrm{r}} \mathrm{r}^{2} \cdot \theta-\mathrm{K}_{\mathrm{r}} r^{2} \theta_{0}=\frac{\mathrm{Mr}^{2}}{2} \cdot \ddot{\theta}
\end{gathered}
$$

From Eq. (11), the natural frequency can be obtained, as defined by Eq. (12).

$$
w_{n}=\sqrt{\frac{k_{r}}{\frac{M+2 m}{2}}}
$$

Initially, this prototype would be represented by a model with two degrees of freedom of the system, which comprises a tangential force to the pulley that gives rise to torsion and bending of the shaft, as shown in Fig. 4. The vibration analysis with two degrees of freedom defines a relationship between the displacement torque $x_{t}(t)$ and bending $x_{f}(t)$. However, such gages have the characteristics to assess normal strain along its longitudinal center line. Considering the way, the SGs were positioned in the shaft, and interconnected with $\mathrm{WB}$, it is possible to analyze the torsional deformations independently of the flexional ones. Because of this setup, the analysis with one degree of freedom for each shape deformation (torsion/flexion) was accessible, as performed in this paper. This paper studies only the influence of torsional strain.

In this study, we adopted the logarithmic decrement, which is the result of a single pulse imposed on the system (free vibration). The term logarithmic decrement refers to the logarithmic reduction rate related to the reduction of movement after the boost, as the energy is transferred to other parts of the system or is absorbed by the element itself. In this oscillating system with damping, which is excited by an impulse, its answer comes in the form of decay in time. It is noteworthy that at $t=t_{m}$, the system response is denoted by $y_{m}$, and the response at time $t=t_{n}+2 \pi r / w_{d}$, where $r$ is the frequency ratio and $w_{d}$ is the natural damped frequency, is denoted by $y_{m}+i$. Thus, the logarithmic decrement $(\delta)$ can be obtained from two measurements $y_{m}$ and $y_{m+i}$, in the free motion regime, defined by Eqs. (13) and (14).

$$
\begin{gathered}
\delta=\frac{1}{n} \cdot \ln \left(\frac{y_{m}}{y_{m+i}}\right)=\frac{2 \pi \cdot \zeta}{\sqrt{1-\zeta^{2}}} \\
\zeta=\frac{\delta}{\sqrt{\delta^{2}+(2 \pi)^{2}}}
\end{gathered}
$$

where, $n$ is the peak order of the oscillations. Considering the geometric relationship between the displacement $x(t)$ and the torsion angle (angular displacement) $\theta+\theta_{0}(t)$, shown in Fig. 5, it was established the balance equation without the influence of forcing. Considering that the shaft is clamped at one end and that it is depicted in equilibrium, it satisfies the equation of motion (15), shown below.

$$
\left(\frac{M+2 m}{2}\right) \ddot{x}+c \dot{x}+k_{r} x=0
$$

The behavior described by the system (Figs. 3 and 5) depends on the solution of the differential Eq. (15). A

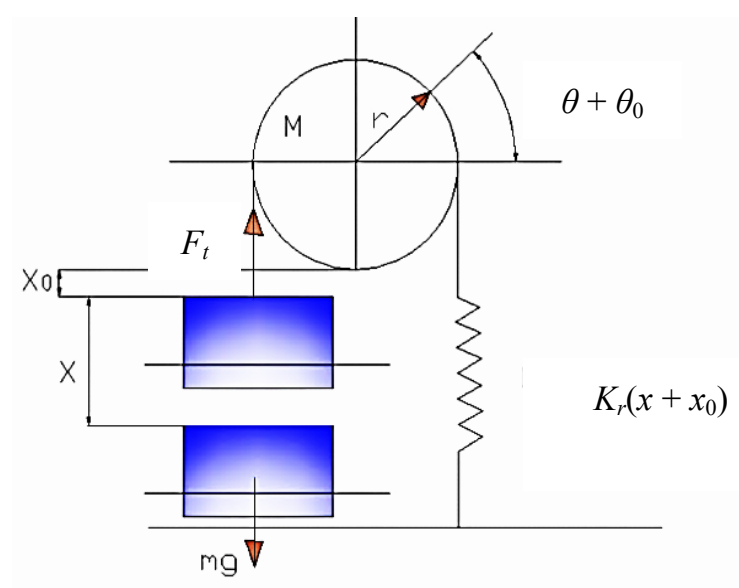

Fig. 5 Model form twisting movement. 
general solution is shown in Eqs. (16) and (17). The damping ratio $\zeta$, defined in Eq. (14), satisfies the condition $0<\zeta<1$.

$$
\begin{gathered}
x(t)=\text { A.e } e^{-\zeta w_{n} t} \sin \left(w_{d} \cdot t+\Phi\right) \\
w_{d}=w_{n} \sqrt{1-\zeta^{2}}
\end{gathered}
$$

$\omega_{d}$ stands for the damped natural frequency, $w_{n}$ represents natural frequency (Eq. (12)), and the integration constants $\mathrm{A}$ and $\Phi$, are given by equations in Ref. [12].

The camshaft is subjected to a dynamic torque. In this case, the angular speed of the camshaft is assumed to be constant and the movement is examined under this assumption. Considering the Euler equation and $x_{0}$ $=0$, set forth in Eq. (15), the results for an underdamped system with one degree of freedom with force $F^{\prime}(t)$ is represented by the differential Eq. (19), whose solution is introduced by Eq. (20), with the condition exhibited in Eq. (18).

$$
\begin{aligned}
& \left(\frac{M}{2}+m\right)=m^{\prime} \\
& m^{\prime} \ddot{\theta}+c \dot{\theta}+K_{r} \theta=F^{\prime}{ }_{t} \cos w \cdot t_{\mathrm{c}} \\
& x(t)=\mathrm{Ae}^{-\zeta \mathrm{w}} \mathrm{n}^{\mathrm{t}} \sin \left(w_{d} t+\Phi\right)+\mathrm{X} \cos (w \cdot t-\theta)
\end{aligned}
$$

where, $M$ is the pulley mass, $m$ is the shaft mass, $m$ ' is the final mass and $\mathrm{A} X, \Phi$ and $\theta$ are integration constants (forced system).

For underdamped forced movement with a sinusoidal force with amplitude $f_{0}$, the classic equations of movement are as shown in Eqs. (21) to (24).

$$
\begin{gathered}
\mathrm{X}=\frac{f_{0}}{\left(w_{n}^{2}-w^{2}\right)^{2}+\left(2 \cdot \zeta w_{n} \cdot w\right)^{2}} \\
\Phi=\tan ^{-1} \frac{w_{d}\left(x_{0}-\mathrm{X} \cos \right)}{v_{0}+\left(x_{0}-\mathrm{X} \cos \theta\right) \zeta w_{n}-\mathrm{w} \cdot \mathrm{X} \cdot \sin (\theta)} \\
\theta=\tan ^{-1} \frac{2 \zeta w_{n} w}{\left|\left(w_{n}^{2}-w^{2}\right)\right|}
\end{gathered}
$$

$$
\zeta=\frac{c}{c_{c r}}=\frac{c}{2 \cdot m^{\prime} \cdot w_{n}}
$$

where, $\zeta$ is the damping ratio, defined in Eq. (24), $c_{c r}$ is the critical damping coefficient and $\omega_{n}$ stands for the natural frequency of vibration of the prototype.

The frequency ratio $\left(r^{\prime}\right)$ can be experimentally determined considering the condition established by Eqs. (25) and (26).

$$
\begin{aligned}
\frac{\mathrm{d}}{\mathrm{d} r}\left(\frac{\mathrm{Xk}}{\mathrm{F}}\right)=\frac{\mathrm{d}}{\mathrm{d}_{r}}\left\{\left[\left(1-r^{2}\right)^{2}+(2 \zeta r)^{2}\right]^{-\frac{1}{2}}\right\}=0 \\
r^{\prime}=\sqrt{1-2 \zeta^{2}}=\frac{w}{w_{n}}
\end{aligned}
$$

In a forced system, resonance occurs when the excitation frequency of system becomes closer (or equal) to the system natural frequency $\left(w=w_{n}\right)$. This condition is established in underdamped systems because for low damping there is a frequency band around the natural frequency at which the dynamic amplification values are high. Therefore, this region may occur with large vibration amplitude even when the amplitude of the exciter forces is small. For excitation frequencies close to zero $\left(r^{\prime} \cong 0\right)$ system behavior is of the static type. For frequencies far above the natural rate $\left(r^{\prime}>1\right)$, there is a great reduction in the vibration amplitude. The result of the torsional strain in the analysis of simulation allows the shaft to be considered in its totality. Vibrational setting values for the variables are indicated in Table 2 .

In the stepped shaft, shown in Fig. 6, the modeling is done via Eqs. (5) and (6), whose parameters were already discussed. Eq. (27) is a general equation to obtain the distortion

$$
\gamma(t)=\frac{\frac{x(t)}{r} \cdot G \cdot\left(2 \frac{r_{6}}{J_{6}}+\frac{r_{5}}{J_{5}}\right)}{\left(2 \cdot \frac{l_{4}}{J_{4}}+\frac{l_{5}}{J_{5}}\right)}
$$

\section{Results}

The analytical modeling was based on the solution of 
Table 2 Parameters and constants vibration.

\begin{tabular}{lll}
\hline Parameters & $\begin{array}{l}\text { Without } \\
\text { forcing }\end{array}$ & $\begin{array}{l}\text { With } \\
\text { forcing }\end{array}$ \\
\hline$w_{n}(\mathrm{rad} / \mathrm{s})$ & 57.7 & 46.8 \\
$W(\mathrm{rad} / \mathrm{s})$ & 10.9 & 11.7 \\
$W_{d}$ & 57.2 & 46.1 \\
$c_{\text {crit }}$ & 417 & 285.4 \\
$K r(\mathrm{~N} / \mathrm{m})$ & 17,603 & 17,603 \\
$f_{n}(\mathrm{~Hz})$ & 9.2 & 6.2 \\
$K_{\text {res }}(\mathrm{N} / \mathrm{m})$ & 8,406 & 8,406 \\
$f(\mathrm{~Hz})$ & 1.74 & 1.86 \\
$T(s)$ Teórico & 0.57 & 0.51 \\
$r$ & 0.19 & 0.25 \\
$c(\mathrm{~N} / \mathrm{s})$ & 58.2 & 58.4 \\
$\zeta$ & 0.13 & 0.17 \\
$r p m$ & 105 & 112 \\
$x(\mathrm{~m})$ & 0 & \\
$V_{0}(\mathrm{~m} / \mathrm{s})$ & 0 & \\
$G\left(\mathrm{~N} / \mathrm{m}^{2}\right)$ & $8.0 \times 10^{9}$ & \\
$E\left(\mathrm{~N} / \mathrm{m}^{2}\right)$ & $19.5 \times 10^{10}$ & \\
\hline
\end{tabular}

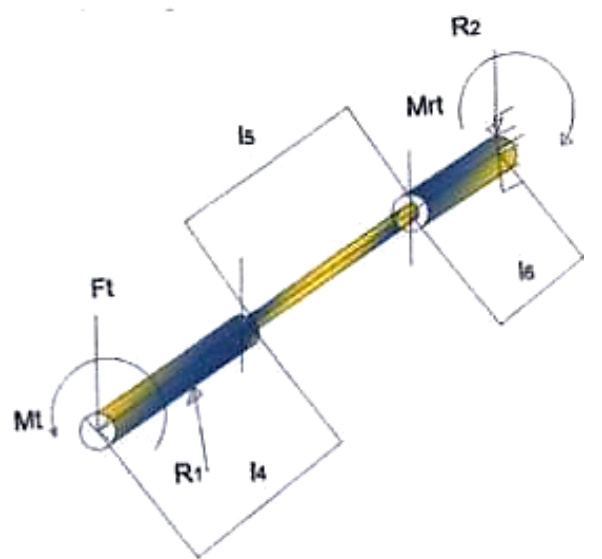

Fig. 6 Stepped shaft details.

differential Eqs. (15) and (19), considering the physical constants of Tables 1 and 2, which contains the vibrational parameters. These amounts are used in the already mentioned Matlab program in order to generate the graphics that allow the comparison among the experimental and analytical results. The experimental analysis was established in a continuous cycle, shown in Fig. 7. Strain configuration for the free underdamped motion was experimentally obtained. Fig. 7 exhibits the graph of strain over time. An initial forced period of $0.09 \mathrm{~s}$ is visible, after which the signal reaches a plateau during $0.13 \mathrm{~s}$. Thus, free oscillation time of $0.29 \mathrm{~s}$ takes place, corresponding to a frequency of $3.4 \mathrm{~Hz}$, exposing natural angular frequency of $21.7 \mathrm{rad} / \mathrm{s}$, with three representative periods (non-zero amplitude) of twisting. Finally, one can see a final free damped oscillation with period equal to $0.42 \mathrm{~s}$ within a total cycle of $0.51 \mathrm{~s}$, which corresponds to a complete rotation of the cam.

For the forced damping movement, using a complete cam, a complete rotation cycle was established with a period of $0.56 \mathrm{~s}$, shown in Fig. 8 . This condition has been reproduced several times yielding a new torsion with the same period until the end condition is established. The experimental analysis showed a frequency of $2.17 \mathrm{~Hz}$ and $105 \mathrm{rpm}$ for the electric motor.

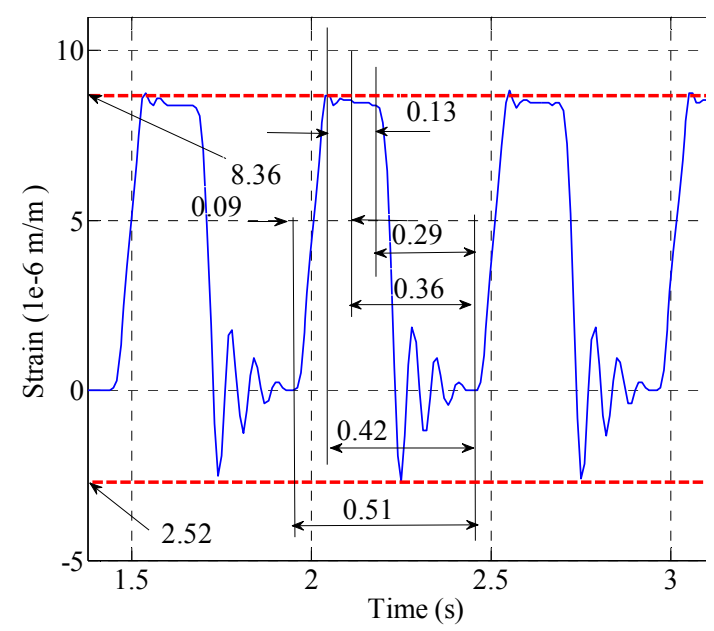

Fig. 7 Strain graph obtained experimentally by free damped oscillation of the shaft, without forcing.

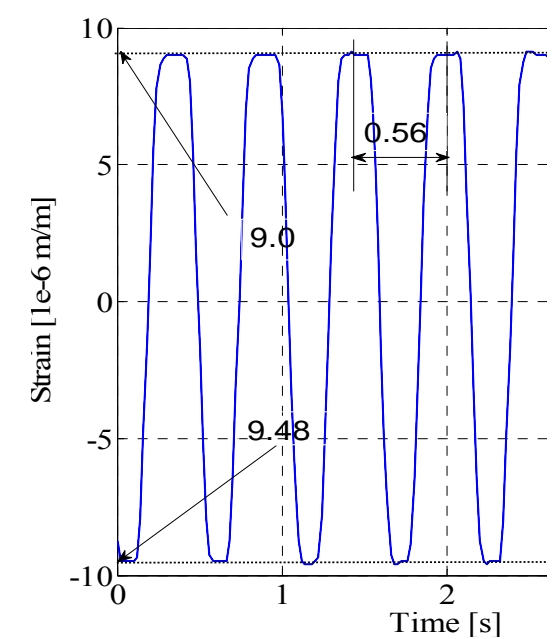

Fig. 8 Strain graph obtained experimentally by forced damped oscillation of the shaft. 


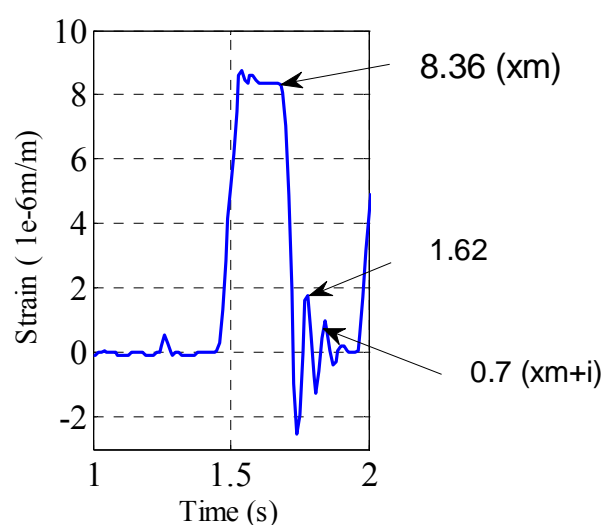

Fig. 9 Detail of Fig. 7 between 1.0 and 2.0 s. These data were used to experimentally obtain the logarithmic decrement $(\delta)$.

The logarithmic decrement $(\delta)$, formerly defined in Eqs. (13) and (14) is depicted in Fig. 9.

This logarithmic decrement $(\delta)$ is a very important parameter in order to qualify the damping coefficient of the prototype.

In Fig. 10, the analytical study (full line) is now compared to the experimental one (dashed line). Comparing the free oscillatory regime, the analytical result has a period of $0.46 \mathrm{~s}$ while the experimental one has a period of $0.42 \mathrm{~s}$ in the same regime, yielding a discrepancy of $9 \%$.

Fig. 11 introduces the analysis in the time domain, for the forced oscillation. Monitoring the torsional vibration frequency, one can notice the experimental period of $0.56 \mathrm{~s}$ and an analytical period of $0.57 \mathrm{~s}$. They show an excellent agreement, yielding a discrepancy of roughly $2 \%$.

Fig. 12 shows both analytical (dashed line) and experimental (full line) study of shaft. During the period without forcing, the strain is concentrated in the central axis of a spiral, whose limits are established by the value of strain variation produced by the experimental forcing system.

Fig. 13 establishes shaft torsional vibration analysis, based on time domain, which exhibits the standard behavior of the prototype.

In this work, the FFT (fast Fourier transform) algorithm was used to change the signal in the time domain to the frequency domain. One can see in Fig. 14 a characteristic frequency behavior presenting a peak. As the prototype changes in time or damages with expected use, it will change both time and frequency domain characteristics.

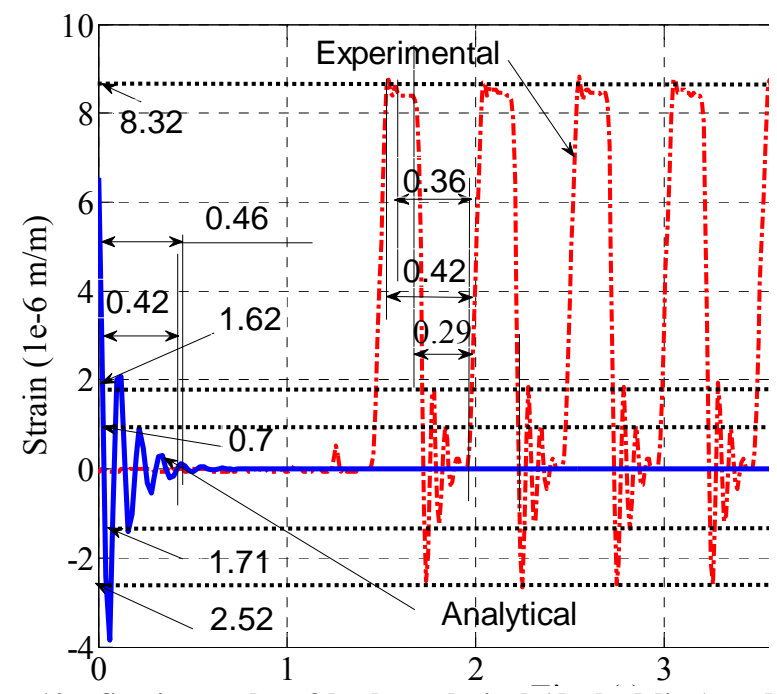

Fig. 10 Strain results of both analytical (dashed line) and experimental (full line) study without forcing.

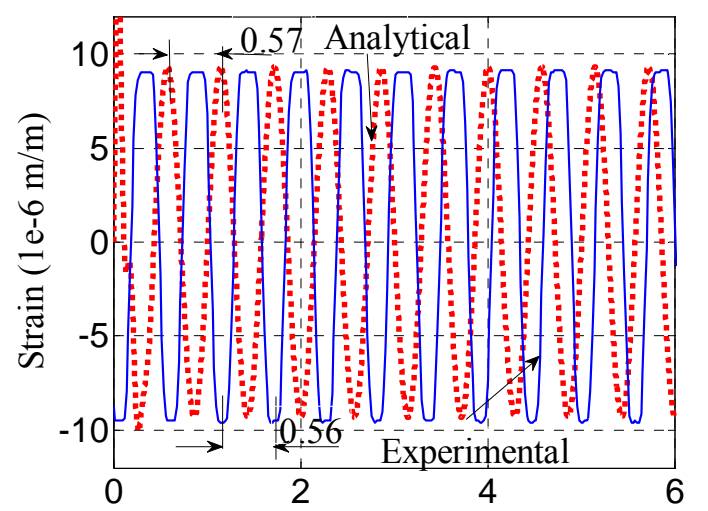

Fig. 11 Strain results of both analytical (dashed line)and experimental (full line) study with forcing.

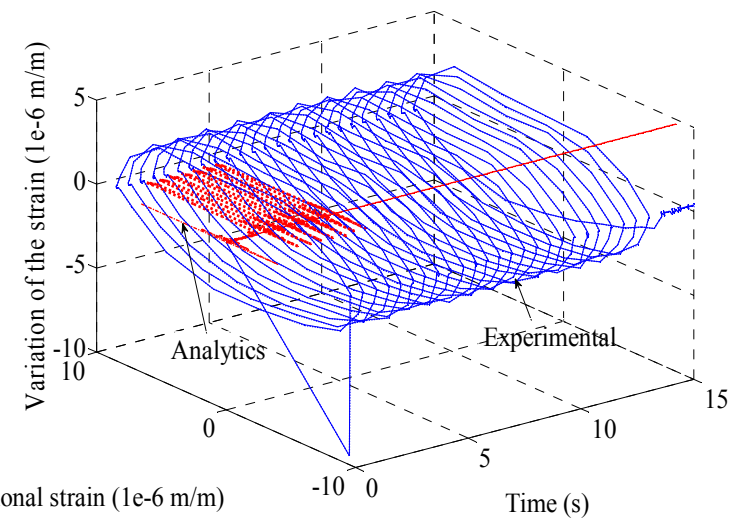

Fig. 12 Strain variation spiral graph over time exhibiting, at its central axis, torsional vibration obtained by analytical and experimental studies. 


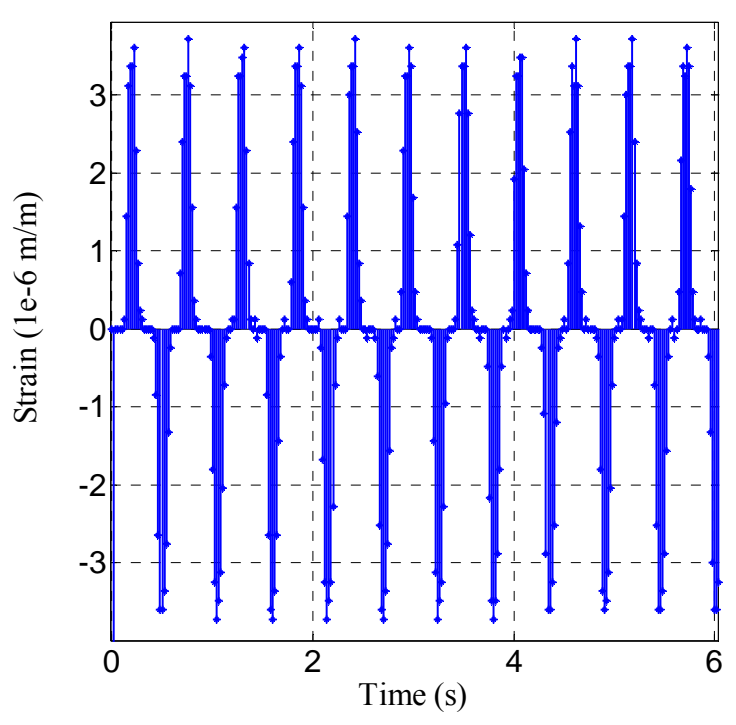

Fig. 13 Torsional vibration analysis in time domain.

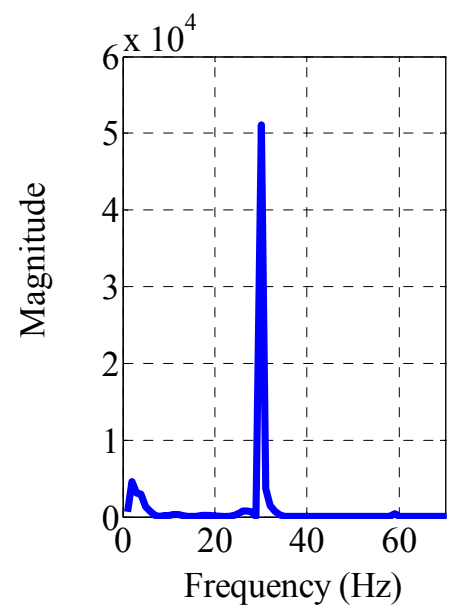

Fig. 14 Torsional vibration analysis in frequency domain.

\section{Conclusions}

In this work, both analytical and experimental modeling of torsional vibration was accomplished. In order to do this study, a prototype was designed and manufactured. The way the strain gauges were fixed in the half section of prototype ( $1 / 2 \mathrm{WB})$ made possible to analyze the shaft torsion in its whose length. The cantilever setup in only one side of the prototype shaft and the swing length caused a different deformation condition for the torsional moment, produced in free form and by ramming. Comparing both analytical and experimental results, a great accuracy was obtained, what strongly suggests that this prototype represents a satisfactory representation of harmonic cyclic movement. This behavior qualifies the prototype for studies in system stability as well as signal sensitivity predictions of the analytical model for shaft torsions.

In this study, our prototype has undergone a loading with two degrees of freedom. The way the SGs were used in this prototype, allowed us to analyze the loadings separately, replacing the more complex system by two systems each one of one degree of freedom. This approach demonstrates the superiority of our setup for studying more complex systems.

As Xiang [9], presented by your article and referenced by Figs. 8a, 8b and 9 on the accuracy and consistency of its torsional vibration analysis results in the field of time and frequency, based on the principles of laser Doppler velocimetry. This article also shows results as Figs. 13 and 14 in relation to spectral diagram of torsional vibration of the shaft about the time domain and frequency, which are obtained by the application of a further analysis based on deformation of the shaft produced by a single component of the multiaxial effort. The employee cams eccentricity to the shaft, to generate vibration which turns its natural frequency, causing various components $W_{n i}$ of natural frequency $W_{n}$ (calculated and presented in Table 2). These results demonstrate the major vibration component peak of the field frequency $\left(W_{n i}=30 \mathrm{~Hz}\right.$, Fig. 14). All the natural frequency peaks of the components are different of the peak excitation frequency $(W), \mathrm{r}^{\prime}<1$. These results demonstrate the quality and consistency of the analysis of the prototype.

\section{References}

[1] $\mathrm{Wu}$, J. J. 2007. "Torsional Vibration Analyses of a Damped Shafting System Using Tapered Shaft Element." Journal of Sound and Vibration 306: 946-54.

[2] Chen, Y. Z. 2001. "Torsional Free Vibration of a Cylinder with Varying Cross-Section and Adhesive Masses." Journal of Sound and Vibration 241 (3): 503-12.

[3] Castro, L. R., Vie' Villeb, P., and Lipinskia, P. 2006. "Correction of Dynamic Effects on Force Measurements Made with Piezoelectric Dynamometers." International Journal of Machine Tools \& Manufacture 46: 1707-15. 
[4] Quin, J., Yu, D., Liu, Y., Wang, G., and Cai L. 2006. "Low Frequency Torsional Vibration Gaps in the Shaft with Locally Resonant Structures." Physics Letters A 348: 410-5.

[5] Gaspari, D., and Aristodeno, M. 2005. "Torsional and Flexure Analysis of Orthotropic Beams by a Boundary Element Model." Engineering Analysis with Boundary Elements 29: 850-8.

[6] Huang, D. G. 2007. "Characteristics of Torsional Vibrations of a Shaft with Unbalance." Journal of Sound and Vibration 308: 692-8.

[7] Han, Q., Zhao, J., and Chu, F. 2012. "Dynamic Analysis of a Geared Rotor System Considering a Slant Crack on the Shaft." Journal of Sound and Vibration 331: 5803-23.

[8] Saka, Z., and Yilmaz, Y. 1992. "Torsional Vibrations of
Shafts." Mech. Mack Theory 27 (3): 225-33.

[9] Xiang, L., Yang, S., and Gan, C. 2012. "Torsional Vibration Measurements on Rotating Shaft System Using Laser Doppler Vibrometer." Optics and Lasers in Engineering 50: 1596-601.

[10] Salarieh, H., and Ghorashi, M. 2006. "Free Vibration of Timoshenko Beam with Finite Mass Rigid Tip Load and Flexural-Torsional Coupling." International Journal of Mechanical Sciences 48: 763-79.

[11] Roukema, J. C., and Altintas, Y. 2006. "Time Domain Simulation of Torsional-Axial Vibrations in Drilling." International Journal of Machine Tools \& Manufacture 46: 2073-85.f

[12] Inmam, D. J. 2001. Engineering Vibration. 2nd edition. New Jersey: Prentice Hall International. 